\title{
Criminologie
}

\section{Une approche de la criminalité féminine à travers l'exemple du hooliganisme}

\author{
Dominique Bodin, Luc Robène, Stéphane Héas et Martin Gendron
}

Volume 38, numéro 2, automne 2005

La police en pièces détachées

URI : https://id.erudit.org/iderudit/012668ar

DOI : https://doi.org/10.7202/012668ar

Aller au sommaire du numéro

\section{Éditeur(s)}

Les Presses de l'Université de Montréal

ISSN

0316-0041 (imprimé)

1492-1367 (numérique)

Découvrir la revue

Citer cet article

Bodin, D., Robène, L., Héas, S. \& Gendron, M. (2005). Une approche de la criminalité féminine à travers l'exemple du hooliganisme. Criminologie, 38(2), 195-224. https://doi.org/10.7202/012668ar
Résumé de l'article

La question du genre est un fait reconnu depuis très longtemps dans la construction des actes délictueux. Pourtant, dans le cas très précis du hooliganisme, aucune étude ne s'est jamais intéressée à l'existence ou non de femmes hooligans. Est-ce à dire qu'il n'en existe pas ? La réalité sociale du hooliganisme en Europe est bien différente. Il existe des femmes hooligans. Leur négation tient à de multiples raisons : l'absence de traitement de la singularité, les a priori positifs dont jouissent les femmes quant aux comportements violents, l'utilisation d'une définition trop restrictive du hooliganisme qui empêche de comprendre la genèse de certains événements et la place des femmes dans ceux-ci, la difficulté de penser une violence féminine alors que certaines femmes subissent chaque jour de nombreuses formes de violence. À travers l'étude des noyaux durs des groupes de supporters du soccer en France, on observe cependant qu'un certain nombre d'entre elles reconnaissent participer et avoir participé à des actes hooligans. Elles possèdent parfois des rôles et des statuts importants au sein des groupes qui les ont obligatoirement confrontées à la violence. Si, physiquement, elles ne sont pas au coeur des affrontements les plus durs, elles sont parfois les instigatrices ou les égéries de certains groupes pour qui la violence est une partie intégrante et intégrative du supportérisme.
Ce document est protégé par la loi sur le droit d'auteur. L'utilisation des services d'Érudit (y compris la reproduction) est assujettie à sa politique d'utilisation que vous pouvez consulter en ligne.

https://apropos.erudit.org/fr/usagers/politique-dutilisation/ 


\title{
Une approche de la criminalité féminine à travers l'exemple du hooliganisme
}

\author{
Dominique Bodin \\ Laboratoire d'anthropologie et de sociologie (LAS) \\ Université de Rennes 2 \\ dominique.bodin@uhb.fr \\ Luc Robène \\ Laboratoire d'anthropologie et de sociologie (LAS) \\ Université de Rennes 2 \\ luc.robene@uhb.fr \\ Stéphane Héas \\ Laboratoire d'anthropologie et de sociologie \\ Université de Rennes 2 \\ stephane.heas@uhb.fr \\ Martin Gendron \\ Université du Québec à Rimouski \\ Département de sciences de l'éducation \\ martin_gendron@uqar.qc.ca
}

RÉSUMÉ - La question du genre est un fait reconnu depuis très longtemps dans la construction des actes délictueux. Pourtant, dans le cas très précis du hooliganisme, aucune étude ne s'est jamais intéressée à l'existence ou non de femmes hooligans. Est-ce à dire qu'il n'en existe pas? La réalité sociale du hooliganisme en Europe est bien différente. Il existe des femmes hooligans. Leur négation tient à de multiples raisons: l'absence de traitement de la singularité, les a priori positifs dont jouissent les femmes quant aux comportements violents, l'utilisation d'une définition trop restrictive du hooliganisme qui empêche de comprendre la genèse de certains événements et la place des femmes dans ceux-ci, la difficulté de penser une violence féminine alors que certaines femmes subissent chaque jour de nombreuses formes de violence. À travers l'étude des noyaux durs des groupes de supporters du soccer en France, on observe cependant qu'un certain nombre d'entre elles reconnaissent participer et avoir participé à des actes hooligans. Elles possèdent parfois des rôles et des statuts importants au sein des groupes qui les ont obligatoirement confrontées à la violence. Si, physiquement, elles ne sont pas au cœur des affrontements les plus durs, 
elles sont parfois les instigatrices ou les égéries de certains groupes pour qui la violence est une partie intégrante et intégrative du supportérisme.

MOTS CLÉS • Femmes, hooliganisme, genre.

Abstract: For a long time, gender has been recognised as a significant matter in constructing criminal acts. Yet, in hooliganism in particular, no study has ever been focused on whether there are female hooligans or not. Does it mean there aren't any? Socially, European hooliganism is much different in reality: there are female hooligans. They are denied for many reasons: peculiarity has not been studied, positive prejudices are attributed to women when touching on violent behaviours, the definition of hooliganism is too restrictive and hides the origins of events and women's roles in such events, or it is difficult to imagine there are violent women when some of them are the everyday victims of many sorts of violence. By studying the hard cores of French supporters, we observed that a certain number of females admitted they were taking part or had taken part in hooligan acts. Sometimes they played significant roles and status within the groups and it inevitably brought them to violence. Physically, they were not at the heart of the hardest clashes, but they may have been the inciters or the driving forces of the groups for which violence is an integral and integrating part of supporterism.

KEY WORDS • Female, hooliganism, gender.

\section{Introduction}

\section{Comprendre le hooliganisme}

Comprendre le hooliganisme, ses origines et les formes qu'il emprunte, oblige à dépasser les définitions habituelles, les représentations collectives, les stéréotypes et les préjugés qui bien souvent prévalent en la matière, limitant de fait son interprétation. Ainsi, traditionnellement, dans l'imaginaire collectif, le hooligan est de nationalité anglaise. Il est jeune, de sexe masculin, mal inséré socialement, délinquant dans la vie ordinaire, imbibé d'alcool et prend prétexte du match de soccer pour venir commettre ses méfaits dans le stade. La réalité sociale s'avère être cependant plus complexe. Car, contrairement à ce que révèle le sens commun, ce phénomène n'est pas plus réductible aux seuls Anglais qu'il n'est imputable aux délinquants ordinaires: le hooliganisme concerne essentiellement pour ne pas dire exclusivement le soccer et ce, quel que soit le pays ou le continent ${ }^{1}$ (Bodin, 1999a; 2003a), agrégeant une

1. En 1997 , le plus important quotidien sportif français, L'Équipe, dénombrait ainsi plus de 1300 morts dus aux actes de hooliganisme dans les stades de soccer du monde entier. Nous pouvons ainsi prendre quelques exemples de ces violences: 
diversité d'expériences et de parcours qu'il convient d'objectiver. Il existe bien quelques affrontements lors des matches de basket-ball en Grèce ou en Turquie, pendant certaines rencontres de cricket en Inde, ou encore dans le cadre d'activités plus variées comme ceux qui, durant la finale du championnat d'Europe de water-polo le 15 juin 2003, ont opposé les supporters croates à ceux de la Serbie et Monténégro, offrant un exemple de l'extension du phénomène. Lors de cette finale, les supporters croates ont lancé des insultes antiserbes qui ont entraîné des violences que rarement des sports autres que le football avaient connues : jets de bouteilles, affrontements avec des barres de fer, attaques des supporters adverses avec des fusées de détresse, etc. Les conflits ethniques peuvent ainsi perdurer et prolonger la guerre dans le sport qui devient un terrain idéal pour la construction ou le renforcement des identités nationales (Grubisa, 2003). Mais ces manifestations de violence restent cependant sporadiques. Comprendre le hooliganisme ne saurait donc se réduire au simple constat de faits qui, parfois d'une extrême violence physique, ne constituent que la surface visible du phénomène, les mécanismes fondamentaux du mouvement se situant plus en profondeur. C'est pourquoi il apparaît indispensable de resituer les actes et les comportements des hooligans dans une dynamique historique et sociale pour tenter de les analyser en privilégiant une approche compréhensive. Une approche qui tente d'articuler dynamiques collectives et trajectoires singulières redonnant une place légitime à la complexité des expériences vécues par les acteurs.

Très souvent pour ne pas dire trop souvent le hooliganisme est appréhendé et caractérisé dans ses variances à partir de son expression finale, la violence. Celle-ci peut revêtir différentes formes et investir divers modes d'affrontements: la violence physique ou la dégradation

- 1964 à Lima (Pérou), un but refusé lors d'un match opposant le Pérou à l'Argentine, match qualificatif pour les Jeux olympiques, provoque une bagarre générale: 328 morts, 500 blessés,

- 1967 à Kayseri (Turquie), un but contesté provoque des bagarres lors d'un match de championnat: 41 morts, 600 blessés,

- 1979 à Rome (Italie), au stade olympique un supporter de la Lazio, Vincenzo Paparelli, est tué par un projectile tiré du virage opposé par un tifosi de la Roma,

- 1982 à Moscou (URSS), les faits seront rendus publics huit années plus tard, la répression d'une manifestation de supporters du Spartak, à la suite d'un match de coupe de l'UEFA qui opposait l'équipe moscovite à celle de Haarlem (Pays-Bas), a provoqué la mort de 340 personnes,

- 1995 à Bruges (Belgique), avant le quart aller de la coupe des coupes qui opposait Bruges à Chelsea (Angleterre), un supporter belge est poignardé par un Anglais. 
de biens pouvant être exercées soit entre groupes de supporters dans le stade, soit à l'encontre des forces de l'ordre, soit encore contre des passants sans rapport apparent avec le soccer ou même le supportérisme. Elle peut aussi conduire à la destruction de voitures, de vitrines, au « caillassage $^{2}$ » de bus ou à la détérioration de wagons, etc. Or cette catégorisation du hooliganisme à partir de ses types de production, pour objective qu'elle puisse sembler se révéler, n'indique en aucune façon la manière dont des individus, bien souvent ordinaires, en arrivent à commettre pareils actes. Il n'y a là qu'un constat amenant à considérer les violences sous le seul angle du passage à l'acte ou de la transgression réprimée de normes établies, ce qui revient à limiter leur acception à une définition sociologique traditionnelle du crime (Durkheim, 1895).

Une telle approche révèle par contraste les biais qualitatifs qu'elle est supposée réduire. En effet, comment échapper au caractère a priori sexué du hooliganisme dès lors qu'est retenue pour unique définition du phénomène, celle de la violence physique la plus dure, matérialisée par le sang versé et la destruction des biens? Si la validité de la statistique criminelle est depuis toujours passionnément débattue (Cusson, 1989; Roché, 1994; Robert, 1994; Fillieule, 2001, entre autres), la question du genre est un fait reconnu depuis longtemps dans la construction des actes délictueux (Chesnais, 1981).

\section{De la place des femmes dans les études sur la criminalité}

Or, aucune étude, quel que soit le champ disciplinaire considéré, n'aborde la question de la participation des femmes dans le hooliganisme. D’une manière plus générale, quel que soit le pays également, la criminalité féminine reste moins étudiée que celle des hommes.

Une des raisons tient peut-être au fait que les femmes sont proportionnellement toujours moins souvent mises en cause que leurs homologues masculins, et ce, quelles que soient les catégories d'infraction. À titre d'exemple, et sans entrer dans le détail de la construction des statistiques

2. Une des actions favorites des supporters hooligans consiste à attendre l'arrivée des bus de supporters adverses, parfois très loin du stade au-dessus des ponts autoroutiers ou du périphérique (voie rapide qui ceinture Paris) par exemple, afin de les bombarder de projectiles en tous genres (cailloux, boulons, etc.). 
criminelles de ces différents pays ${ }^{3}$, les femmes représentaient, au titre de l'année 2002, $23 \%$ des personnes mises en cause aux États-Unis, un peu plus de $15 \%$ au Canada et $14,2 \%$ en France.

Cependant, une première remarque s'impose. Alors que d'une manière générale le taux global de criminalité, tous crimes et délits confondus, tend à diminuer dans ces trois pays (Gannon, 2001; Ouimet, 2002; Roché, 2003; Wallace, 2003) la proportion des femmes incriminées reste stable.

Une seconde remarque concerne la délinquance juvénile impliquant des filles. Bien que stable depuis bientôt 6 ans, celle-ci avait fortement progressé au cours des décennies précédentes dans tous les pays précités.

Le désintérêt pour la criminalité féminine tient peut être également au fait que les femmes sont moins souvent que les hommes impliquées dans les crimes et délits les plus violents. Ainsi, la délinquance chez les filles est considérée comme relativement rare et moins grave que chez les garçons (Simourd et Andrews, 1999). La visibilité sociale, la dangerosité et le sentiment d'insécurité qui sont associés à la criminalité masculine, qu'elle soit juvénile ou non, suscitent davantage d'études et de recherches.

La distinction ne s'arrête cependant pas là. Si aujourd'hui les études sur la délinquance féminine et masculine tendent à se confondre dans leurs démarches et dans la nécessaire prise en compte d'approches multifactorielles, elles ont fait à l'origine l'objet d'un traitement différentiel. Très souvent, les premières études sur les femmes délinquantes insistaient essentiellement sur leurs problèmes de personnalité (Giordano, 1978, par exemple), alors que la délinquance masculine était dans le même temps, étudiée sous l'angle de facteurs extrinsèques comme la pression du groupe de pairs, l'exclusion sociale, l'inégalité ou la vulnérabilité sociale, etc. Intériorisation et personnalisation de l'acte d'un côté, par rapport à l'extériorisation et la collectivisation de l'autre. Quand bien même la délinquance féminine est étudiée, le dimorphisme est tel que la délinquance masculine reste souvent présente en creux.

Comme le suggèrent Dauphin et Farge (1997: 11), les représentations féministes ont probablement contribué pour un temps à neutraliser le thème de la violence des femmes, car «comment penser la

3. Pour des comparaisons plus en profondeur et consulter les rapports annuels en détail, consultez: l'Uniform Crime Report sur <http://www.fbi.gov> (États-Unis), le Code criminel sur < http://www.msp.gouv.qc.ca $>$ (Canada) et les Crimes et délits constatés sur <http:// www.interieur.gouv.fr $>$ (France). 
violence des femmes alors que la violence sur les femmes est de loin la plus manifeste»? La violence féminine est alors une sorte d'«impensable culturel» à révéler. C'est pourtant autour de cette question que s'est articulée concurremment à partir de la fin des années 1970 une critique féministe de la criminologie à travers, par exemple, les travaux de Bertrand $(1979 ; 2003)$ et de Parent (1998). Bertrand (2003) montre, notamment, que nombre d'études relatives à la criminalité féminine ont été empreintes, jusque dans les années 1980, de représentations et préjugés relatifs à la place et à l'image de la femme dans la société et le droit. Parent (1998), quant à elle, met en évidence l'influence des études féministes dans la compréhension d'une étiologie distincte de celle construite par le regard des hommes.

L'analyse des actes hooligans est en tout point conforme aux actes de délinquance «ordinaire». Faut-il pour autant en déduire qu'il n'existe aucune femme hooligan? Ou bien encore, faut-il comprendre que, si ces femmes ne participent pas directement aux affrontements les plus violents pour des raisons «physiques", leur rôle dans l'émergence et l'accomplissement des violences hooliganistiques se situent dans un ailleurs qu'il s'agit de trouver? Enfin, à considérer qu'il existe effectivement des femmes hooligans : comment comprendre le déni de reconnaissance qui participe à rendre socialement et culturellement invisible leur participation aux actes délictueux? Autrement dit, dans quels types d'enjeux s'inscrit la relégation, voire la négation du hooliganisme féminin?

\section{Matériel et méthodes}

Cet article tente de répondre à ces questions à partir d'une enquête à la fois qualitative et quantitative menée de 1995 à 2002 (Bodin, 1998; $1999 b$; 2001). Le hooliganisme est généralement le fait d'un nombre limité de supporters rassemblés dans ce qu'il est convenu de dénommer le «noyau dur» du groupe. Ce noyau est composé essentiellement des leaders et des individus présents à tous les matches de championnat, que ce soit à domicile ou en déplacement. Les listes des membres du noyau dur étaient fournies par les responsables des associations de supporters. Notre présence dans le stade, au sein du groupe nous permettant de vérifier la participation active des individus interviewés au noyau dur. Ont ainsi été interrogés 530 membres des noyaux durs des clubs de supporters par questionnaires (436 hommes et 94 femmes) sur les clubs de Bordeaux, Marseille, Paris, Toulouse, Rennes, Nantes et Lyon pour 
le soccer de première division, et 167 membres, par entrevues, dont 15 femmes ${ }^{4}$. Celles-ci sont toutes célibataires et aucune n'a fait l'objet de condamnation pour hooliganisme.

Trois méthodes de collectes de données ont été utilisées:

- les questionnaires, comprenant 34 sujets, remplis par les membres des noyaux durs des clubs de supporters visant à connaître leurs modes de sociabilité, leurs habitudes, leurs réseaux d'amitié et d'inimitié, leur participation à la violence, ont été analysés à l'aide du logiciel Sphinx Lexica millenium $\AA$;

- des entretiens semi directifs de type histoires de vie (Becker, 1986; Bertaux, 1997) visant à recueillir l'histoire vécue par chacun, à retrouver les lignes de vie, à articuler les domaines d'existence et voir s'il existait dans ces histoires singulières des récurrences et des homologies structurales (Demazière et Dubar, 1997). Les extraits retranscrits ici mettent en évidence ces homologies et ont été choisis sciemment en fonction de leur pertinence, mais aussi de l'appartenance des femmes à des groupes distincts afin de montrer que ces homologies ne représentent pas un trait culturel de leur groupe d'appartenance;

- des observations pour déterminer les modalités de pratiques, mais aussi pour confirmer, infirmer ou compléter les «dires» recueillis dans les deux premières méthodes par les «faits».

Il s'agit d'une enquête d' «hooliganisme autorévélé », les individus interrogés répondant positivement à la question: «Avez-vous déjà participé à des actes de hooliganisme (bagarres, destructions de matériel)?» et étaient invités à accorder, s'ils le désiraient, un entretien complémentaire. Les entretiens ont été réalisés de préférence en dehors du local des supporters pour ne pas que les interviewés soient influencés par la présence de leurs pairs.

\section{Résultats et discussion}

\section{Hooliganisme et sexe}

Si les réponses à la participation aux actes hooligans semblent montrer le caractère particulièrement sexué de cette forme de violence, cette observation mérite cependant d'être nuancée.

4. Des données plus vastes (Bodin, 2003a), il ressort que 70,5\% des hooligans sont membres des noyaux durs des groupes de supporters, $23 \%$ sont simplement membres de ces mêmes communautés, les $6,5 \%$ venant au stade seuls ou avec des amis. 
TABLEA U 1

Participation aux actes hooligans en fonction du sexe ${ }^{5}$

\begin{tabular}{|l|c|c|c|}
\hline $\begin{array}{c}\text { Sexe } \\
\text { Affrontement }\end{array}$ & Supporters féminins & Supporters masculins & Total \\
\hline Oui & $27,7 \%(26)$ & $62,2 \%(271)$ & $82,3 \%(297)$ \\
\hline Non & $72,3 \%(68)$ & $37,8 \%(165)$ & $17,7 \%(233)$ \\
\hline Total & $\begin{array}{c}100 \%(94) \\
(\mathbf{1 7 , 7} \%)\end{array}$ & $\begin{array}{c}100 \%(436) \\
(\mathbf{8 2 , 3} \%)\end{array}$ & $\mathbf{1 0 0 \% ( 5 3 0 )}$ \\
\hline
\end{tabular}

La dépendance est très significative. $\mathrm{chi}^{2}=37,35, \mathrm{ddl}=1,1-\mathrm{p}=>99,99 \%$.

Les femmes reconnaissant participer aux actes de hooliganisme ne représentent que $8,8 \%$ du total des individus, ce qui, à première vue, apparait comme très nettement inférieur aux pourcentages de femmes mises en cause pour des faits similaires dans la société civile. Elles représentent, en effet, 14,2\% en 2002 (ministère de l'Intérieur, 2002). Le caractère sexué du hooliganisme doit cependant être discuté. Tout d'abord, les femmes dans la société civile constituent à peu près $50 \%$ de la population alors que les femmes dans l'échantillon ne représentent que $17,7 \%$ de l'ensemble des répondants. Les femmes «violentes» sont en fait statistiquement surreprésentées tout à la fois par rapport à l'échantillon $(27,7 \%$ reconnaissent avoir participé à des affrontements contre 17,7\% de répondants), mais également au regard de leur proportion dans le public dans les stades. Selon des enquêtes précitées (cf. Matériel et méthodes), réalisées selon un échantillonnage "par unité type» (Chauchat, 1985), le public des stades de soccer en France est composé de 86,9\% d'hommes et de 13,1\% de femmes.

À l'image des travaux de Blumstein et al. (1986), une analyse fine requiert de distinguer deux dimensions : la participation et la fréquence. Alors que, toutes formes de violences confondues, le rapport de participation est de 1 à 10 entre femmes et hommes, celle-ci augmente avec la gravité ou la dangerosité des infractions commises. Pour les infractions les plus graves (coups et blessures volontaires ayant entraîné des

5. Les valeurs du tableau sont les pourcentages en colonne établis sur 530 observations. Ce tableau est construit sur la strate de population «suporters du soccer» contenant 530 observations et définie par le filtrage suivant : Sport $=\{$ Soccer $\}$ et supportérisme $=\{$ oui $\}$. 
blessures) le rapport est de 1 à 13,4. L'écart de fréquence réduit considérablement puisqu'elle n'est que 1,8 fois supérieure chez les hommes. Une première lecture simple, pour ne pas dire simpliste, consisterait à expliciter cette différence par le nécessaire engagement physique dans les violences les plus importantes, ce que nous discuterons à l'aide des entretiens.

Il faut également prendre en compte le fait que la quasi-totalité des femmes qui reconnaissent participer aux actes de hooliganisme sont membres des groupes de supporters, ce qui représente 25 personnes, parmi lesquelles 19 sont membres des noyaux durs, la dernière venant au stade avec son ami, membre d'un groupe de supporters.

\section{Hooliganisme, sexe et condamnations}

Dernière observation générale concernant les hooligans, aucune femme n'a, à ce jour, fait l'objet d'une quelconque condamnation dans le cadre de la loi Alliot-Marie de 1995 relative à la sécurité dans les enceintes sportives. Toutefois, constater cette absence de délit caractérisé revient en définitive à observer simplement qu'aucune femme ne s'est fait prendre. Tout comme il convient aussi de remarquer plus largement que seulement 37 condamnations ont été prononcées depuis l'application de la $\operatorname{loi}^{6}$. Ce qui est fort peu compte tenu du nombre d'incidents, souvent très violents, qui émaillent chaque rencontre de première division tous les week-ends, au point que, selon le rapport confidentiel des renseignements généraux en date du 17 décembre 2001, intitulé «Hooliganisme, la violence supportériste», loin d'être endigué par les dispositifs sécuritaires et législatifs, le phénomène tend à prendre de l'ampleur.

On serait donc tenté de penser qu'il n'existe pas de femmes réellement violentes parmi les supporters. Mais il faut prendre en considération un certain nombre de facteurs. Tout d'abord, le fait que les femmes délinquantes semblent traitées différemment des hommes délinquants par la police et les tribunaux. Même si les études sont loin d'être unanimes sur cette question, il existerait selon certains auteurs un «effet de chevalerie » qui fait qu'à acte équivalent de gravité, les femmes sont condamnées moins sévèrement que les hommes, voire pas du tout (Heidensohn, 1997; Fillieule, 2001). Le hooliganisme n'échappe pas à

6. Source: Direction des affaires criminelles et des grâces. 
la règle. Les femmes hooligans sont considérées comme ne pouvant pas être à l'origine ou participer aux actes les plus violents. Les jeunes femmes, outre l'éducation reçue et le contrôle social plus important dont elles sont l'objet pourraient avoir, dans le hooliganisme, comme le suggèrent Gottfredson et Hirschi (1990) pour la délinquance, un «contrôle de soi» plus fort en moyenne que les garçons, ce qui les prémunirait contre les actes les plus graves. Beaucoup des événements impliquant les femmes ont lieu souvent très loin des périmètres de sécurité et des forces de l'ordre, comme le «caillassage» des bus adverses, par exemple. Il faut également se souvenir que, bien souvent, les personnes condamnées le sont pour des prétextes futiles, comme le fait de faire pénétrer des fumigènes dans l'enceinte du stade dans le cas de L. T., leader du club des supporters marseillais des Yankees en 2002, ou pour la simple raison qu'ils ont transgressé de manière trop visible, donc objectivement indéfendable, les interdits et la relative mansuétude des dirigeants et des forces de l'ordre. Constatons également, comme dans le cas des incidents opposant les supporters du PSG (Paris-Saint-Germain) à ceux de Galatasaray en 2001, qui firent 56 blessés dont 7 graves, lors d'un match en Ligue des champions, que les arrestations avérées sont souvent peu nombreuses (11 individus) pour des raisons inhérentes à la philosophie des forces de l'ordre. Celles-ci préferent souvent assurer une gestion «au mieux de l'intérêt général», entre la stricte application de la loi Alliot-Marie et les complications apportées par le contrôle d'une foule de 20 à 40000 personnes entrant ou sortant de stades implantés, pour la plupart en France, en centre-ville. D’où ce choix délibéré que, plutôt d'interpeller les contrevenants et provoquer des mouvements massifs de contestation, de les accueillir dans le stade en les maintenant sous surveillance, mais surtout de les accueillir sous peine de voir les incidents se dérouler en ville (Bodin, 1999a; Bodin et Trouilhet, 2001). Enfin, le traitement du hooliganisme, comme la délinquance ordinaire, est sans doute sexuellement différencié et différentiel: les femmes pourraient ainsi bénéficier d'a priori positifs les excluant de toute responsabilité dans les faits les plus graves en raison de leur «faiblesse supposée». De nombreux travaux généralistes (Becker, 1986; Robert et al., 1994; Chesnais, 1981; Roché, 2003) ou spécialisés (Bodin, 2001; 2003a) ont montré les différences de traitement existant. Le nombre de condamnations n'est donc vraisemblablement pas un indicateur suffisamment opérant pour révéler et quantifier à lui seul l'existence du hooliganisme ni pour en distinguer a fortiori les formes sexuées. 
Ces quelques données demandent de toute évidence à être complétées par le vécu de celles qui participent aux actes de violences afin de passer du «statut» à «l'expérience» (Elias et Dunning, 1986). Si les chiffres avancés répondent "partiellement» à la question de savoir qui est violent, ils ne restituent pas davantage la dynamique de la violence qu'ils ne nous permettent de comprendre la place particulière que peuvent éventuellement tenir les femmes dans ces violences.

\section{Reconsidérer la définition du hooliganisme permet de faire émerger le rôle des femmes}

Ce qui importe en premier lieu est de reconsidérer la définition du hooliganisme. D'abord en prenant en compte la dimension dynamique du phénomène, ensuite en révisant le balisage du champ des violences que son exercice suppose. À l'image des travaux de Skogan (1990), Roché $(1996$; 2001), Debarbieux $(1996$; 2002) consacrés à d'autres domaines des conduites agonistiques, il ne semble en effet guère possible de continuer à considérer la violence des supporters sous le seul angle du résultat final. Résultat qui dans sa version la plus abrupte, celle d'un supporter anglo-saxon poignardé au début de l'année 1998 dans une ruelle, semble être au sport ce que l'homicide volontaire et prémédité est au code pénal. C'est également dans l'enchaînement successif de faits en apparence plus ou moins dérisoires (vols d'insignes ou d'emblèmes, insultes et provocations, culture des groupes de supporters, etc.) qu'il faut aller chercher la genèse d'événements beaucoup plus dramatiques et inquiétants. Les violences décrites précédemment ne sont en fait qu'un «accomplissement pratique ${ }^{7} »$, aboutissement d'un long processus fait d'interactions sociales subtiles et complexes entre les différents acteurs du spectacle sportif (supporters, dirigeants, policiers, journalistes, etc.). La complexité du phénomène doit donc être prise dans sa dynamique d'ensemble: le moindre petit fait, les incivilités, les invectives, pouvant avoir des effets de spirale sur la violence. Par ailleurs, se pose le problème de la nature des violences prises en compte. Car restreindre l'observation des actes à la seule violence physique exclut mécaniquement une large palette de facteurs explicatifs comme la «tchatche ${ }^{8} »$, les joutes oratoires, la provocation ou encore l'historicité

7. Garfinkel (1967).

8. Provocations verbales et rituelles dans le langage argotique des supporters. 
des antagonismes qui précèdent bien souvent les rixes et les bagarres. Facteurs dont l'importance en tant que catalyseur ou déclencheur est aujourd'hui reconnue dans bien d'autres domaines de la délinquance juvénile (Lepoutre, 1997; Duret, 1999; Wieviorka, 1999; Roché, 2001).

En Grande-Bretagne, dès 1978, Marsh critiquait cette conception trop réductrice du hooliganisme. Car se cantonner à la violence physique et nier les violences morales et symboliques revient en fait à nier la portée de dispositifs structurés remarquables comme l' «aggro», mise en scène ritualisée de la violence destinée à impressionner l'adversaire. Or le jeu qui consiste à impressionner, à paraître fort et dangereux, à chercher à faire peur aux autres, supplante pourtant bien souvent le passage à l'acte. Le basculement ne se produit en définitive que lors de la transgression des règles tacites de l' «aggro» (attaquer une fille par exemple ${ }^{9}$ ) ou à la suite de l'intervention des forces de l'ordre. Certes, la frontière entre violences physiques, symboliques ou morales, est parfois ténue (Héas et Bodin, 2001; Bodin et al., 2004). Sans doute parce que les violences considérées demeurent consubstantielles les unes des autres, qu'elles ne représentent, en définitive, qu'un moment ou une étape dans les processus sociaux et qu'elles «alimenteront, exacerberont ou renouvelleront en spirale d'autres expressions de violence» (Wieviorka, 1999: 17). Cependant, prendre en considération l'ensemble de ces étapes ou de ces articulations permettrait, par effet de contraste, de rendre visible une catégorie d'acteurs, parmi lesquels sans doute un certain nombre de femmes, pour qui l'expression de la violence emprunte des chemins détournés ou en tout cas dont l'activité violente échappe à une économie statistique rigide enfermée dans le comptage strict du «violemment physique et visible». C'est la raison pour laquelle s'impose ici un véritable changement de focale sociologique. Car en recourant à une acception plus large de la violence, de ses logiques de progression spiralaires et de ses catalyseurs, il devient en effet possible de cerner plus facilement la part potentielle des femmes dans l'émergence et la construction d'un processus dynamique. Il devient possible de comprendre en quoi leur action participe des faits les plus bénins à l'émergence de faits plus graves.

9. Accord tacite particulièrement révélateur de la culture genrée du soccer. 


\section{De la place des femmes dans les violences}

Si les femmes semblent a priori absentes des grands affrontements, elles en sont néanmoins souvent les témoins ou parfois les victimes:

Tu sais, nous, on participe que très rarement aux bagarres. On fait pas le poids. On peut pas aller à la baston comme eux contre les autres garçons. Des fois, on est obligée de se défendre quand même quand on est coincée. On fait ce qu'on peut mais ce n'est pas notre rôle. Si. Des fois on fait comme les garçons, on attend les autres, on caillasse les bus, il m'est arrivé aussi de tout casser à la sortie d'un stade avec les autres mais c'est tout. (Femme, 20 ans, membre du groupe des Boulogne Boys de Paris.)

Cet extrait d'interview met bien en lumière la différence d'engagement qui distingue hommes et femmes dans la participation aux affrontements. La dangerosité des événements constitue le premier critère d'appréciation. Aux garçons, la violence physique, les filles participant pour leur part à une violence plus «distante» ou à des destructions qui ne les confrontent pas directement aux supporters adverses. Ce que semble confirmer cet autre entretien:

Tu sais alors que je suis dans un groupe violent, certains disent même qu'on est les plus violents en France, j'ai été prise dans des bagarres. Mais nous, les filles, on participe pas généralement, on laisse les garçons, car des fois ça dégénère et nous, physiquement, on peut pas faire face aux garçons. Parfois eux ils se battent à coup de poings, de matraques ou de battes. Bon nous on provoque plutôt, des fois on balance des trucs sur les supporters adverses, mais souvent on est chargée de protéger le matériel. (Femme 19 ans, membre du groupe des South Winners de Marseille.)

Outre la dangerosité des affrontements et l'utilisation d'armes, ces entretiens laissent de surcroît percevoir que les femmes peuvent jouer un rôle spécifique dans les actes de violence.

\subsection{Rôles et statuts des femmes dans les groupes de supporters}

Si la femme supporter semble bien éviter l'affrontement physique direct, certaines héritent cependant d'un rôle très important: celui de responsable des emblèmes et des insignes (bâches et drapeaux aux couleurs et armoiries du groupe). Rôle primordial, car ces attributs ont bien évidemment un coût, mais ils ont de surcroît un sens social très important 
puisqu'ils signent, signifient et authentifient la présence du groupe dans le stade aux supporters adverses. «Bâcher» chez l'adversaire, c'est-à-dire se rendre dans le stade adverse afficher le nom de son club de supporter est vécu par les deux parties en présence comme une provocation. La détention de ces emblèmes oblige donc très souvent les femmes à subir ou à être confrontées à la violence :

Moi, j'ai jamais vraiment cherché la bagarre parce que nous c'est pas possible contre les garçons. Alors nous, on nous confie les bâches et les banderoles, on doit les rentrer dans les stades et les garçons eux ils sont là pour veiller au grain. Une fois, on n'était pas assez nombreux on s'est fait agresser et piquer une banderole. Ouais il a fallu se défendre, les mecs en face n'ont pas hésité à nous taper dessus pour l'avoir. (Femme, 19 ans, membre des Bad Gones de Lyon).

La détention et la responsabilité des emblèmes n'est cependant pas un rôle anodin, car au-delà du coût que représente ce matériel, c'est l'honneur du groupe de supporters qui est en jeu dans le fait de «bâcher» chez l'autre ou de se faire voler les insignes du groupe. Chaque groupe possède son musée où sont exposés les trophées dérobés lors des multiples escarmouches avec les policiers et les supporters adverses (casques de CRS, insignes, Bombers, écharpes, bâches, etc.). Au match suivant, les bâches et emblèmes dérobés seront exhibés dans les tribunes, par provocation, mais aussi par esprit de domination de l'autre, à l'image des seigneurs d'autrefois qui arboraient les armoiries de ceux qu'ils avaient vaincus et vassalisés. Un effet de spirale s'instaure ainsi progressivement. $\mathrm{Au}$ match retour, les supporters tenteront de se déplacer plus nombreux, pour venger l'affront, pour montrer leur puissance. Ceux qui se sont fait voler une bâche ou des emblèmes importants viendront en force, éventuellement armés, pour les reprendre et restaurer leur groupe dans sa légitimité et son intégrité initiales, sa grandeur symbolique.

Cette observation renvoie à la définition culturaliste du statut et du rôle: «Un rôle représente l'aspect dynamique du statut. L'individu est socialement assigné à un statut, lui-même lié à d'autres statuts. Quand il met en œuvre les droits et les devoirs qui constituent le statut, il remplit un «rôle» (Linton, 1936: 138). À partir de cette définition, il est aisé de comprendre qu'un rôle aussi important que la responsabilité des attributs du groupe qui engage l'honneur, l'existence et l'identité de la communauté toute entière, ne peut être confié qu'à des personnes qui possèdent un statut important au sein de cette entité. Ainsi émerge progressivement 
de l'ombre l'importance du statut de celles à qui sont confiés les rôles de passeurs et de protecteurs des emblèmes de la tribu. Là n'est pourtant pas la seule responsabilité que les femmes exercent. En effet, plus proche encore de la violence, l'une d'entre elles est chargée chez les Ultramarines bordelais de filmer les affrontements avec les supporters:

Moi, mon rôle c'est de filmer les bagarres avec les autres supporters, quand on y va et comment ça se passe. De filmer tout ce que je peux. Après, au local, on se repasse les films et on voit comment on était organisé, on en discute. Et puis aussi, cela sert aussi aux jeunes à leur montrer comment ça se passe. Ça fait partie de la culture du groupe. On leur montre comme ça qu'on n'est pas des peureux. Ça les oblige à faire pareil (Femme, 21 ans, membre des Ultramarines de Bordeaux).

À la lecture de cet entretien, hormis la prise en compte d'un nouveau rôle, on peut observer tout à la fois le caractère prémédité des violences ainsi que leur intégration dans la culture du groupe. Culture d'une certaine forme de violence structurée et structurante, qui participe à «l'instruction» des «nouveaux». La violence fait bien partie intégrante du supportérisme.

Enfin, très souvent ce sont les femmes qui introduisent dans le stade les objets interdits (matraques télescopiques, fumigènes, stylos fusées, etc.). Plusieurs raisons à cela. Tout d'abord, les supporters masculins sont davantage surveillés, épiés, fouillés, car soupçonnés d'être des hooligans potentiels. On peut parler ici de «délit de sale gueule» à moins que cela ne corresponde aussi tout simplement à l'incorporation par les forces de l'ordre de l'un des préceptes de la domination masculine: celui de la virilité et de la violence (Bourdieu, 1998). Une seconde raison tient à l'organisation, ou tout au moins au défaut d'organisation des services de sécurité privés ou policiers qui assurent le filtrage à l'entrée des stades ou du périmètre de sécurité. Une femme ne peut, en effet, faire l'objet de palpation ou de fouille que de la part d'une femme. Or cellesci sont souvent trop peu nombreuses dans les divers services de contrôle et ne peuvent assurer pleinement leur mission qui consiste en définitive, le plus souvent, à des palpations sur quelques personnes choisies de manière aléatoire. Enfin, la fouille, qui permettrait de détecter davantage d'objets interdits, ne peut être effectuée que par des personnes assermentées. La palpation (surfaçage rapide du corps), parce qu'elle se montre relativement inefficace, permet très souvent d'introduire l'attirail nécessaire à l'activité du supporter. 
Ce rôle particulier des filles, passeuses d'armes, véritables « chevaux de Troie» n'est ni nouveau ni original. Les femmes du FLN (Front de libération nationa $1^{10}$ ) durant la guerre d'Algérie, ou leurs homologues vietcong durant la guerre du Vietnam n'agissaient pas différemment. Ces faits nous aident en revanche à mieux comprendre de quelle manière, à travers la violence des antagonismes et les tactiques déployées pour dominer l'interdit et les adversaires, en instrumentant en quelque sorte leur féminité et en adaptant à leur façon ce recours aux failles du système, «les femmes accèdent peu à peu au rang de sujet dans la guerre » (Branche, 2002). Sans doute, ce constat n'invalide-t-il pas le problème de la domination et des positions de genre dans la mise au point de ces stratégies. Mais, pour le moins, les statuts et les rôles des femmes hooligans au sein de leurs propres groupes fondent alors une légitimité dans l'action que seule la considération de normes plus étendues dans le processus de construction du hooliganisme et dans la nature des violences considérées peut aider à éclairer et du même coup à reconnaître. Cette reconnaissance dans et par la violence, ajoutée à d'autres regards, à d'autres reconnaissances, montre enfin, au-delà de tout jugement, au-delà de toute considération axiologique, de quelle manière s'effrite progressivement le modèle rassurant de l'invisibilité féminine dans l'histoire des hommes (Thébaud, 1992).

\subsection{Noyau dur, femmes et hooliganisme}

Ainsi, au chahut (Testanière, 1967), au désordre et au tumulte apparents des tribunes répond en fait une organisation très hiérarchisée d'où ne saurait être totalement exclue la présence active des femmes. Les diverses études relatant la composition des groupes font apparaître une unité de structuration quels que soient l'époque et le lieu (Zimmerman, 1987; Ehrenberg, 1991; Dupuis, 1993a/b; Mignon, 1993; Bromberger, 1995 ; Roumestan, 1998, etc.). Dupuis (1993a : 135-137) distingue trois entités: «les meneurs, leur nombre varie entre cinq et dix personnes», ce sont les responsables, les leaders du groupe, souvent fondateurs de celuici, «le noyau dur». Il peut compter de dix à deux cents membres [...] Ils sont présents à presque chaque match à domicile et effectuent au

10. Mouvement algérien visant la libération de l'Algérie de l'occupation française dans les années 1950-1960. 
minimum tous les déplacements à hauts risques. Agressifs en paroles et en actes, ils sont souvent responsables des incidents » et enfin, la masse du groupe des supporters, "les stagiaires aussi appelés satellites, suiveurs ou figurants [...] La masse des stagiaires est beaucoup plus différenciée que le noyau dur. On y trouve aussi bien des adolescents de treize ou quatorze ans, qui veulent jouer au casseur, que des quadragénaires en mal de sensations fortes». Les membres du noyau dur des clubs de supporters sont effectivement ceux qui ont une expérience du supportérisme inscrite dans le temps. Ils sont les plus assidus et les plus expérimentés et sont généralement présents à tous les matches que ce soit à domicile ou en déplacement. La structuration est conforme à celle observable en France. Le hooliganisme est, par voie de conséquence, le fait d'un nombre limité de supporters: le noyau dur du groupe, composé essentiellement des leaders et des individus présents à tous les matches de championnat que ce soit à domicile ou en déplacement.

Mais comment intègre-t-on le noyau dur dont les membres sont reconnaissables dans bien des stades à leur position centrale au sein du groupe et au port d'insignes particuliers et plus ou moins visibles, comme c'est le cas chez les South Winners marseillais où seuls les membres du noyau dur arborent le bombers (doublure orange) visible?

La réponse est souvent fuyante et ambiguë : «en faisant ses preuves!». Les Winners distinguent pour leur part l'inscription de l'intégration, c'est-à-dire le fait d'être membre (payer sa cotisation) et le fait d'être accepté et reconnu comme un élément valable à qui il est possible de faire confiance. Certains évoquent même alors, dans ce groupe, comme dans d'autres, des rites initiatiques et des rites intégratifs, les premiers marquant l'entrée dans la communauté, les seconds l'adoption et la réception par les autres membres, à titre de pair. Car l'intégration est double puisqu'il s'agit bien d'un acte délibéré et rationnel d'un individu attiré par les valeurs et l'esprit d'un groupe particulier et qu'il existe également l'autre versant, celui de la communauté qui accepte ou non de recevoir ce nouveau membre et qui reconnaît en lui un individu capable de partager les idéaux et les valeurs du groupe. À ce stade, il y un choix délibéré de l'individu d'abandonner une partie de luimême au profit des valeurs et de la culture du groupe auquel il aspire. C'est un choix conscient de la part de chacun des nouveaux membres lorsqu'il veut intégrer le groupe au sein du noyau dur. Les autres éléments peuvent toujours participer aux activités annexes du groupe, aux tifos (spectacle organisé avant ou pendant le match par les supporters 
des clubs de football) et déplacements, mais ne seront pas reconnus comme étant des membres ou des initiés. Il ne s'agit rien d'autre que $\mathrm{du}$ fonctionnement habituel des «sociétés secrètes» (Simmel, 1908; Martin Saint-Léon, 1901). Le poids du secret est omniprésent, et ce n'est qu'en interprétant les dires et les faits, les non-dits et les actions observables, en croisant les discours des membres des différents groupes les uns sur les autres que l'on peut analyser l'importance des violences dans les sous-cultures déviantes. Le secret est d'ailleurs un élément intégrateur, qui permet de gagner la confiance des autres membres et de protéger le groupe. Pour être intégrés, les nouveaux membres doivent participer activement au fonctionnement de la communauté ainsi qu'aux rites intégratifs qui comportent de nombreuses épreuves agonistiques ${ }^{11}$ : participer à des affrontements divers, à des expéditions punitives, mais aussi voler des insignes, un drapeau ou encore aller saccager le local des supporters adverses en déplacement. Il n'est guère anodin de relever la manière par laquelle tout ou partie de ces épreuves participent à la démonstration du courage et de la virilité intégrant plus largement, comme la force dans les anciens rites du monde ouvrier, une forme d'apprentissage des rôles masculins (Roynette, 2002). Ce qui rend par contraste plus saisissant le modelage qui s'opère alors dans le champ du féminin. Car les femmes qui appartiennent au noyau dur et qui y possèdent un statut et un rôle particuliers ont nécessairement satisfait aux rites agonistiques. Il est, en effet, inconcevable, dans un milieu aussi fortement hiérarchisé, dans lequel la domination masculine est si prégnante, que des femmes puissent faire partie des initiées, de celles sur lesquelles le groupe peut compter, sans qu'elles aient participé réellement et physiquement à des actes de violences de diverses natures. Un seul entretien d'un membre masculin du noyau dur des South Winners relate assez précisément cette question et met en lumière la manière par laquelle, en termes de représentation, ces femmes semblent devenir «des hommes comme les autres», conformément au modèle rousseauiste de l'assimilation de l'humain (universel) à l'homme (masculin) (Théry, 2000):

Ouais, chez nous il y a des femmes dans le noyau dur. Quelques-unes, pas nombreuses. Celles qui y sont ont fait leur preuve comme nous. On les considère comme nous. Elles vont à la baston comme nous s'il le faut. (Homme, 23 ans, membre des South Winners de Marseille)

11. Mélangeant compétition, défi et violence. 


\section{Propos confirmés par les femmes:}

Y a pas de différence avec les garçons. Si tu veux faire partie du noyau dur, t'as pas le choix, tu fais tes preuves comme eux. C'est quoi faire ses preuves? Moi, c'est simple, ils m'ont demandé d'aller casser avec les autres le local des Bordelais quand on s'est déplacé et puis de ramener des trucs, des écharpes, etc., enfin des trucs qui appartenaient aux autres quoi! (Femme, 19 ans, membre des Indians de Toulouse.)

Bon quand j'ai intégré le groupe, en fait, ils m'ont dit que si je voulais continuer avec eux il fallait que je fasse comme eux dans tous les cas. J'ai participé à des expéditions punitives contre les UB 90 de Strasbourg. On est allé rechercher la banderole qu'ils nous avaient prise. On a tout cassé dans leur local et on a ramené des trophées. C'était notre mission. C'est comme ça que t'es acceptée si tu fais ce qu'on te dit et que tu fais tes preuves. (Femme, 20 ans, membres des Devils de Bordeaux.)

À ce stade, il n'est plus possible de nier la participation des femmes dans les actes hooligans les plus violents ni même la rationalité de leurs actes. À l'instar de l'analyse finaliste de Cusson (1981), les actes hooligans poursuivent quatre grands types de fins: l'action, l'appropriation, l'agression et la domination sans que rien dans les faits ne puisse dissocier les finalités masculines et féminines.

Par ailleurs, ne peut-on considérer que la violence des femmes est ici également une réponse à la domination masculine laquelle les conduit à faire, aussi bien, voire mieux, que les hommes dans le domaine de la violence afin d'être acceptées et reconnues? Dans une perspective similaire, au début du siècle, la reconnaissance des femmes sportives par leurs homologues masculins et leur intégration au cercle très fermé des adeptes et experts dans le domaine des pratiques à haut risque supposant la violence des accidents et de la mort, s'accommodait parfaitement de ce type d'asymétrie, alors que les brevets de pilotes aéronautes, par exemple, comportaient des épreuves bien plus rigoureuses et bien plus difficiles pour les femmes que pour les hommes (Robène, 1996). Comment ne pas prendre en compte une telle contrainte alors que de nos jours encore, la plupart du temps et dans bien des domaines, malgré des discours politiques convenus, les femmes continuent d'être obligées dans et par la société ordinaire à faire mieux ou davantage que les hommes pour accéder aux mêmes postes, rémunérations et reconnaissance sociale? (Davisse et Louveau, 1998) 


\subsection{Les femmes comme égéries de la violence au sein des groupes}

Mais à lire ce qui précède on pourrait supposer que les femmes ne font finalement que subir un ordre inéluctablement établi en fonction des normes masculines et qu'elles n'ont jamais de rôle ou de position spécifiques directement reconnus dans l'émergence ou la progression des violences. Certains témoignages de supporters masculins tendent à montrer le contraire :

Y en a qui sont pires que nous. Parce qu'en fait, elles nous poussent à y aller, elles nous encouragent. Elles nous chambrent si on n'y va pas, si on veut reculer. En fait ouais... comment dire... bon si on veut pas passer pour des lopes devant elles il faut y aller quoi (Homme, 23 ans, membre des Devils de Bordeaux).

Les femmes si elles ne sont pas forcément instigatrices deviennent ainsi parfois incitatrices des violences qui prennent corps et sens dans les rapports ambigus entre les sexes.

Des fois, c'est elles qui provoquent les supporters adverses, qui les chambrent, qui les chauffent bon, chez les mecs c'est vrai que c'est dur de se faire chauffer par une nana. Souvent quand c'est comme ça, ça finit mal, on va à la bagarre parce que, les autres, ils acceptent pas de se faire chambrer par les gonzesses. On a beau leur dire, mais souvent les trucs les plus durs arrivent quand c'est elles qui ont cherché les mecs des autres groupes (Homme, 21 ans, membre des Yankees de Marseille.)

Ces propos ne sont pas sans rappeler ceux de Godineau (1997) sur la violence et la férocité verbale et physique dont pouvaient faire preuve les «tricoteuses» durant la Révolution. Badinter (2003) nous rappelle également que, dans bien des cas (femmes SS durant la Dernière Guerre mondiale, violences ethniques au Rwanda, etc.), sans la participation des femmes il n'y aurait pas eu autant de victimes. Que dire enfin du rôle et de la place des femmes militaires dans les violences avérées perpétrées par certains éléments des troupes états-uniennes dans les prisons irakiennes récemment?

Par la simple force de leur présence, elles obligent parfois les hommes à se surpasser et à prouver, à travers la violence produite, la virilité qu'ils sont censés posséder et qui leur donne valeur au cœur du groupe des individus soudainement mis en compétition sous le regard des femmes. Certaines femmes sont d'ailleurs très conscientes du rôle qu'elles jouent : 
Tout à l'beure tu me disais "nous, on provoque». Qu'est-ce que t'entends par là? Ça, tu sais, c'est compliqué parce que y a pas en fait... comment te dire... y a pas que les supporters adverses qu'on provoque. Eux, on les provoque bien sûr. On les traite de tous les noms, on se moque d'eux, on caillasse les bus, tout quoi. Mais tu sais aussi, on provoque les garçons de chez nous, on les pousse à y aller ou à pas reculer. Et, eux, pour prouver, ils se la jouent et ils continuent ou ils vont à la bagarre pour nous aussi (Femme, 19 ans, membre du groupe des South Winners de Marseille).

Resterait à considérer les raisons pour lesquelles, précisément, le regard des femmes, leur présence ou leurs invectives provocatrices s'inscrivent, de cette manière, dans une logique culturelle qui participe en définitive à construire une partition typée des violences produites: la femme demeurant a priori celle qui avive une tendance supposée «naturelle» des hommes à l'agonistique, à la compétition virile et à la démonstration de la force brute, voire à la guerre. Une telle approche permet en réalité de comprendre combien les violences produites le sont d'une manière générale en fonction de rapports de domination complexes où interagissent le masculin et le féminin, alors que les territoires et la nature des violences varient sensiblement d'un genre à l'autre tout en se déplaçant et en se transformant l'un en fonction de l'autre. Inversement, c'est bien la mise en perspective de violences de nature différentes qui permet alors de rendre visible l'existence des femmes comme actrices potentielles de ces violences, c'est-à-dire, a minima, comme actrice de l'histoire dans un monde posé non plus comme territoire de l'universel masculin mais plutôt comme monde irrémédiablement sexué (Virgili, 2002).

\section{4. "Carrières» d'hommes, "carrières» de femmes: une différence essentiellement temporelle}

Ce qui distingue peut-être encore davantage les femmes des hommes dans le hooliganisme est le temps durant lequel elles vont s'adonner aux actes de violences. Le hooliganisme peut, en effet, être associé à une « carrière $^{12}$ ». L'usage de la violence à long terme distinguant ainsi celui qui ne s'adonne qu'occasionnellement à la violence, et ce, souvent, uniquement en réaction à une menace. Mais analyser la «carrière»

12. Nous écrivons sciemment carrière entre guillemets, n'ignorant pas les controverses qui existent autour de ce terme (Gottfredson et Hirschi, 1990; Cusson, 1983; Kokoreff et Faugeron, 2002). 
délinquante ou la «phase criminelle» (Cusson, 1981) permet d'opposer les comportements féminins et masculins en termes de début (âge auquel on commet son premier acte de hooliganisme), de fin et donc par déduction de durée. Ces trois phases permettent de tenter de répondre à certaines questions: quelles sont les raisons ou les facteurs qui influencent l'entrée dans le hooliganisme? Comment expliquer l'arrêt de ces mêmes actes? Comment expliquer que certaines «carrières» semblent plus longues que d'autres?

En utilisant non plus les classes d'âge ${ }^{13}$, mais l'âge civil déclaré lors des enquêtes, deux observations s'imposent d'emblée:

1. $62,96 \%$ (102 individus) des $17-24$ ans de sexe masculin ont 17 ans et $39,51 \%$ (40) ont 18 ans alors que 93,75\% (15 individus) des $17-24$ ans de sexe féminin ont 20 ans. Dans cette classe d'âge privilégiée de recours aux actes hooligans, les femmes sont un peu plus âgées que leurs homologues masculins.

2. 51,95\% (40 individus) appartenant à la classe d'âge des 25-39 ans ont plus de 30 ans alors que dans cette même classe d'âge, aucune femme n'a plus de 27 ans.

Il semble donc que le début de la «carrière» hooligan soit plus tardif chez les femmes, que cette «carrière» s'arrête plus tôt et conséquemment dure moins longtemps que celle de leurs homologues masculins.

L'explication la plus simple, pour ne pas dire simpliste, pour rendre compte de la différence d'âge dans le début de «carrière» pourrait être le contrôle social plus rigoureux exercé sur les jeunes filles par les parents. Un certain nombre d'entres elles l'évoquent d'ailleurs:

Moi je suis venue au stade tard, $j$ 'avais 19 ans, car mes parents ne voulaient pas me laisser sortir seule avec autant de garçons, partir en déplacement pour aller voir des matches durant deux jours et coucher comme ça dans le bus. Ils ne voulaient pas. (Femme, 20 ans, membre du Commando Ultra de Marseille.)

Une analyse plus fine permet de se rendre compte que la venue au stade est rarement, pour ne pas dire jamais, la conséquence d'un amour effréné de ces jeunes filles pour le soccer. Elles aiment ce sport. Certaines pratiquent le soccer en club. Mais toutes indiquent qu'elles sont

13. Celles-ci avaient été utilisées afin de pouvoir les comparer aux statistiques officielles émanant de l'INSEE (Institut national de la statistique et des études économiques) et du ministère de l'Intérieur en France. 
venues au stade et elles se sont inscrites dans le groupe de supporter parce que leur "petit copain» y allait et en faisait partie. Leur inscription dans le supportérisme est différente de celui des garçons qui, eux, y placent tout à la fois une passion exacerbée pour ce sport et le plaisir d'être au stade entre copains.

Pour ces jeunes filles, le supportérisme et le hooliganisme sont un acte mimétique qui leur permet de faire perdurer la relation qu'elles entretiennent avec le garçon qu'elles accompagnent. Ce disant, nous ne nions absolument pas que certaines puissent trouver tout à la fois dans le soccer mais aussi dans les actes de violences un intérêt ou un plaisir personnel.

L'arrêt suit la même logique. Rares sont les jeunes filles qui continuent à venir au stade lorsqu'elles ont rompu leur relation sentimentale. Certains entretiens en parlent de manière explicite:

Ouais, j'en ai connu quelques-unes qui venaient mais c'était pas tout à fait comme nous. Elles venaient parce qu'elles avaient un copain dans le groupe. Bon, le jour où c'était fini entre eux on ne la revoyait plus. Elles aimaient pas le foot comme nous, quoi. Elles venaient d'abord pour être avec lui. Mais ces filles, elles participaient aux violences? Tu sais quand tu suis l'équipe tout le temps dans un groupe comme le nôtre, t'es obligée de participer. Les filles comme les garçons. (Homme, 29 ans, membres des Yankees de Marseille.)

On n'est jamais nombreuses. T'en as qui viennent d'abord pour être avec leur mec sinon elles ne le verraient pas du week-end. [...] Et elles participent à la violence? Ben oui, c'est pas pour ça qu'elles sont plus tendres. Je dirai même que parfois elles en font plus rien que pour prouver à leur mec et aux autres qu'elles sont dignes d'être là. Mais tu sais ça ne dure jamais très longtemps. Quand c'est fini avec lui, ou qu'elles rencontrent quelqu'un d'autre qui s'intéresse pas au foot elles arrêtent de venir. (Femme, 26 ans, membre des Devils de Bordeaux.)

L'autre raison de l'arrêt de la «carrière » de ces jeunes femmes est leur entrée dans la vie professionnelle et la constitution d'une famille ${ }^{14}$. Les jeunes femmes quittent le domicile parental pour s'installer en couple plus tôt que leurs homologues masculins (respectivement 22 et 27 ans en moyenne en France ${ }^{15}$ ). Or cette période est marquée par le repli intrafamilial (De Singly, 1993).

14. Nous entendons par là l'installation avec un conjoint dans un domicile distinct de celui de leurs parents respectifs sans pour autant qu'il y ait un enfant.

15. Données INSEE (2002). 
Reste la question du rapport à la violence et de l'influence du groupe de pairs. Les femmes semblent moins sensibles aux prescriptions, aux pressions et à l'influence du groupe de pairs. À ce titre, peuton réellement parler de groupe de pairs ici dans la mesure où il est masculin dominant? Peut-être, tout d'abord parce que leur intérêt premier n'était pas le groupe, mais celui qu'elles accompagnaient. Peutêtre aussi en fonction du plus grand «contrôle de soi» qu'elles ont intégré tout au long de leur éducation comme nous l'avons évoqué précédemment.

Le hooliganisme ne se distingue pas en termes d'âge, de durée et d'influence sociale, des autres formes de violences étudiées dans les pays occidentaux à l'image des travaux de Tarling (1993) ou de Farrington (1986), entre autres. Alors que les garçons trouvent dans le groupe de pairs et dans la violence un moyen et un lieu de construction, de renforcement, et d'affirmation de leur identité à travers une activité passionnante et passionnelle, qui leur offre un espace de convivialité et de liberté, les jeunes femmes doivent au contraire s'en échapper pour construire une identité féminine moins en proie à la domination masculine qui est le fondement et le ciment de ces groupes sportifs et de supporters sportifs (Bromberger, 1995).

Pour toutes ces raisons, et en dehors de critères naturalisant la violence en l'assimilant à la seule force virile représentée par les hommes, la «carrière » violente des femmes dure moins longtemps.

\section{Conclusion}

Dès lors que nous acceptons d'appréhender le plus objectivement possible la participation des femmes aux actes hooligans, comment expliquer que celle-ci soit en définitive passée sous silence? N'est-ce pas tout d'abord dans le rôle social traditionnellement dévolu aux hommes et aux femmes qu'il faut en rechercher les raisons? Ce n'est pas seulement, comme le suggéraient Elias et Dunning (1986) dans le fonctionnement social des individus, sous la forme du lien segmentaire, qu'il faut donc trouver sens, mais dans le rapport implicite entre les sexes. Les hommes sont censés faire preuve de virilité à travers des activités physiques ou sportives plus ou moins violentes tandis que les femmes se réaliseraient plus facilement dans des activités ludiques, esthétiques ou artistiques. Représentations ancrées dans un rapport de domination que la fémini- 
sation des pratiques sportives contredit pourtant aujourd'hui! Cette virilité peut trouver corps et légitimité dans la violence alors que les femmes sont supposées n'être que douceur et miel, du moins plus sociables (Cario, 1999). Cependant, les rôles ne peuvent être à ce point distincts, sauf à naturaliser la violence en la limitant aux seuls individus mâles censés posséder la force physique nécessaire à son expression. Or Margaret Mead a su très tôt montrer combien sur les plans du pouvoir et de la violence le jeu des genres pouvait se révéler autrement plus complexe que ne le laisse supposer l'histoire du monde occidental (Mead, 1993). La «nature féminine» n'apparaît en définitive que comme une construction culturelle, et son corollaire occidental, la faiblesse et la soumission féminine, n'est qu'un élément intégrant un éventail de possibles fondements d'un discours social de l'inégalité (Bard, 1999). «On ne naît pas femme, on le devient» : la formule magistrale de S. de Beauvoir (Beauvoir, 1949) introduit alors à la question du genre, c'està-dire à la "différence des sexes produite par la culture», prélude à l'analyse des partages réels et symboliques entre les sexes et aux résistances au changement que ces partages suscitent (Perrot, 2003). Dans l'imaginaire collectif, la criminalité féminine semble encore se heurter à une fin de non recevoir. De fait, la question des violences des femmes est encore une question qui divise et qui produit en définitive idéologiquement son contraire: l'absolution par nature. Naturellement et culturellement elles seraient étrangères à la violence quand bien même, comme l'observe Bertrand (2003), l'accroissement de la criminalité féminine correspond aux transformations sociales et à l'homogénéisation des rôles dans la société qui traduit tout à la fois un recours plus important à des actes jusqu'alors "réservés aux hommes», mais également par une plus grande visibilité de la violence des femmes sur leurs enfants, par exemple.

Mais pour reprendre les propos de Perrot (1975 : 88) : «Cette indulgence, au fond, n'est-elle pas suspecte? Refuser à la femme sa nature criminelle, n'est-ce pas encore une façon de la nier?» Cependant, si cette reconnaissance pose problème, il faut sans doute aussi bien en rechercher les causes dans le déni pur et simple qu'instrumente une forme de domination, que dans le choix sélectif que chercheurs et chercheuses opèrent dans les objets qu'ils soumettent à l'analyse (Virgili, 2002). Non pas qu'il faille, d'une quelconque manière, se glorifier de la violence et plus particulièrement de celle des femmes, mais ne pas en parler revient à nier cette violence, à la déconsidérer, à la reléguer au rang des violences 
dérisoires et d'une certaine manière à pérenniser une distinction qui n'a de fondement que la domination à laquelle elle fait écho. Il est vrai que dans le hooliganisme, très, pour ne pas dire trop, souvent, les interprétations n'ont été abusivement faites qu'à partir des catégories sociales les plus importantes proportionnellement, niant, de fait, la singularité (Bodin, 1999 ; 2003a). «C'est ce postulat de départ qui domine la plupart des travaux contemporains sur la violence. Au nom de l'asymétrie statistique, la question de la violence et de l'abus du pouvoir féminin ne saurait être posée» (Badinter, 2003: 89).

Il existe cependant une autre raison à cela. Dans un type de discours féministe qui prévaut depuis les années 1960, il semble inconvenant de parler de la violence des femmes tandis que le combat pour la libération des femmes tient depuis toujours dans la dénonciation des violences symboliques (abus de pouvoir, domination), morales (harcèlement moral et sexuel), physiques (violences conjugales, viols) dont sont victimes les femmes quotidiennement. En observant la littérature traitant des femmes depuis cette époque, force est de constater que les travaux parlant des victimes de la domination masculines abondent. Au point que, comme le souligne Badinter (2003: 16), «on s'intéresse moins à celle qui réalise des exploits qu'à la victime de la domination masculine». Ce disant, que les choses soient claires. Nous ne renions et ne sousestimons en aucune manière le fossé statistique qui sépare les femmes victimes des femmes violentes. Mais il faut, néanmoins, malgré un dimorphisme aussi évident, accepter cette réalité sociale: il existe des femmes violentes dans le hooliganisme comme ailleurs (violence familiale sur enfants, femmes meurtrières, jeunes femmes dans des "gangs» en France et pas seulement aux États-Unis, etc.). Or, comme le remarque Virgili (2002: 8), «en rendant visibles les femmes actrices de l'histoire», il faut accepter l'idée que «l'appartenance au camp des victimes ou à la participation à l'émancipation » doive se conjuguer avec l'éclairage plus «problématique» des zones sombres: celles des femmes actrices de la violence ${ }^{16}$. Il est vrai que l'écart statistique est tel qu'il pourrait imposer le silence à, ce qui semble impensable, une réflexion sur la violence des femmes. Il est pourtant nécessaire, pour comprendre certains cheminements dans la violence, de fonder, comme le proposent

16. Fabrice Virgili évoque notamment, à l'égard de l'histoire des femmes dans la Résistance, une histoire toujours absente des femmes engagées dans les violences de la Collaboration. 
Dauphin et Farge (1997: 11) «une réflexion sur la façon différenciée dont, en histoire et aujourd'hui, les sociétés vivent, pensent et imaginent la violence féminine tandis qu'elles exercent simultanément de la violence sur des femmes». Il faut donc accepter que derrière l'universelle domination masculine, puisse exister des lieux possibles et des moments particuliers où s'exercent des violences produites par les femmes. Des violences qui, pour surprenantes qu'elles soient, précisément parce qu'elles sont exercées par des femmes, chose à laquelle nous ne sommes pas habitués, doivent être interprétées et comprises. Faute de quoi le risque est grand d'en faire une réalité sociale éludée, ce qui va à l'encontre des principes mêmes de la sociologie: l'interprétation et la compréhension des mondes vécus.

\section{Références}

Badinter, E. (2003). Fausse route. Paris: Odile Jacob.

Bard, C. (1999). Un siècle d'antiféminisme. Paris: Fayard.

De Beauvoir, S. (1949). Le deuxième sexe. Paris: Gallimard.

Becker, H.-S. (1986). Outsiders, Études de sociologie de la déviance. Paris: Métailié. Bertaux, D. (1997). Les récits de vie. Paris: Nathan université.

Bertrand, M.-A. (1979). La femme et le crime. Montréal: L'Aurore.

Bertrand, M.-A. (2003). Les femmes et la criminalité. Outremont, Québec: Athéna.

Blumstein, A., Cohen, J., Roth, J.-A., \& Visher, C.-A. (1986). Criminal careers and "carreer criminals". Washington: National Academy Press.

Bodin, D. (1998). Sports et violences. Analyse des phénomènes de déviances et de violences chez les supporters de football à partir d'une étude comparative du supportérisme dans le basket-ball, le football, le rugby et le volley-ball. Bordeaux: Thèse université de Bordeaux 2.

Bodin, D. (1999a). Le hooliganisme, Vérités et mensonges. Paris: Esf.

Bodin, D. (1999b). Le hooliganisme en France. Sport, 165/166, 38-118.

Bodin, D. (2001). Les problèmes posés par l'utilisation des statistiques policières et judiciaires comme source d'interprétation sociologique. L'exemple du hooliganisme. Revue juridique et économique du sport, 58, 7-19.

Bodin, D. (2003). Le hooliganisme. Paris: PUF

Bodin, D. \& Trouilhet, D. (2001). Le contrôle social des foules sportives en France: réglementation, difficultés d'application et extension des phénomènes de violences. In D. Bodin (ed.), Sports et violences (147-168). Paris, Chiron.

Bodin, D., Robène, L., \& Héas, S. (2004). Sports et violences en Europe. Bruxelles: Éditions du Conseil de l'Europe.

Bourdieu, P. (1998). La domination masculine. Paris: Seuil. 
Branche, R. (2002). Des viols pendant la guerre d'Algérie. Vingtième siècle, 75 , 123-132.

Bromberger, C. (1995). Le match de football. Etbnologie d'une passion partisane à Marseille, Naples et Turin. Paris: Maison des sciences de l'homme.

Cario, R. (1999). Les femmes résistent au crime. Paris: L'Harmattan.

Chauchat, H. (1995 [1985]). L'enquête en psycho-sociologie. Paris: PUF.

Chesnais, J.-C. (1981). Histoire de la violence. Paris : Robert Laffont.

Cusson, M. (1989). Croissance et décroissance du crime. Paris: PUF.

Cusson, M. (1989 [1981]). Délinquants pourquoi? Paris: Bibliothèque québécoise.

Dauphin, C. \& Farge, C. (1997). De la violence et des femmes. Paris: Albin Michel.

Davisse, A. \& Louveau, C. (1998). Sport, école, société: la différence des sexes, féminin, masculin et activités sportives. Paris: L'Harmattan.

Debarbieux, E. (1996). La violence à l'école, 1. État des lieux. Paris: Esf.

Debarbieux, E. (2002). L'oppression quotidienne, Recherches sur une délinquance des mineurs. Bordeaux: Rapport remis à l'Institut des hautes études de la sécurité intérieure.

Demazière, D. \& Dubar, C. (1997). Analyser les entretiens biographiques, l'exemple des récits d'insertion. Paris: Nathan, collection «Études et recherches».

De Singly, F. (1993). Les rivalités entre les genres dans la France contemporaine. Esprit, 11, 54-64.

Dupuis, B. (1993a). Le hooliganisme en Belgique : histoire et situation actuelle, $1^{\text {re }}$ partie. Sport, 143, 133-157.

Dupuis, B. (1993b). Le hooliganisme en Belgique: histoire et situation actuelle, $2^{\mathrm{e}}$ partie. Sport, 144, 195-226.

Duret, P. (1999). Les jeunes et l'identité masculine. Paris: PUF.

Durkheim, É. (1895). Les règles de la méthode sociologique, Paris: PUF.

Ehrenberg, A. (1991). Le culte de la performance. Paris: Calmann-Levy.

Elias, N. \& Dunning, E. (1994 [traduction de 1986]). Sport et civilisation, La violence maîtrisée. Paris: Fayard.

Farrington, D. P. (1986). Age and Crime. Crime and justice, 7, 189-250.

Fillieule, R. (2001). Sociologie de la délinquance. Paris : PUF.

Garfinkel, H. (1967) Studies in ethnomethodology. Englewood Cliffs, New Jersey: Prentice-Hall, Inc.

Gannon, M. (2001). Crime comparisons between Canada and the United States. Juristat, 21 (11).

Giordano, P. (1978). Girls, Guys and Gangs: The changing Social Context of Female Delinquency. The Journal of Criminal Law and Criminology, 69, 126-132.

Godineau, D. (1997). Citoyennes, boutefeux et furies de guillotines. In C. Dauphi \& A. Farge (ed.), De la violence et des femmes (35-56). Paris: Albin Michel.

Gottfredson, M. R., \& Hirshi, T. (1990). A General Theory of Crime. Stanford: Stanford University Press. 
Grubisa, D. (2003). Le water-polo ou la guerre par d'autres moyens. Courrier international, 660, 14.

Héas S. \& Bodin D. (2001). Football et rugby féminins: quelles violences symboliques? In D. Bodin (ed.), Violences et sports (77-88). Paris: Édition Chiron.

Lepoutre, D. (1997). Cour de banlieue, Codes, rites et langages. Paris: Odile Jacob. Linton, R. (1936). De l’homme. Paris : Éditions de minuit, traduction 1968.

Marsh, P. (1978). Aggro, The illusion of violence. Londres: Dent \& Ltd.

Martin Saint-Léon, E. (1901). Le compagnonnage, son histoire, ses coutumes, ses règlements et ses rites. Paris: Imprimerie du compagnonnage.

Mead, M. (1993). Mours et sexualité en Océanie. Paris : Pocket.

Mignon, P. (1993). La société du samedi: supporters, ultras et hooligans, Étude comparée de la Grande-Bretagne et de la France. France: rapport remis à l'Institut des hautes études de la sécurité intérieure.

Ministère de la Justice français. Direction des affaires criminelles et des grâces. (Document interne).

Ouimet, M. (2002). Explaining the American and Canadian Crime "Drop" in the 1990s. Canadian Journal of Criminology, 44, 33-51.

Parent, C. (1998). Féminismes et criminologie. Bruxelles: De Boeck-Wesmael.

Perrot, M. (1975). Délinquance et système pénitentiaire en France au $19^{\mathrm{e}}$ siècle. Annales Économie, société et civilisation, 1, 67-91.

Perrot, M. (2003: 27 novembre). Les partages des sexes, histoire inachevée. Le Monde.

Robène, L. (1996). Vers la création d'un sport féminin: des filles de l'air aux aéronautes. In P. Arnaud. \& T. Terret, (ed.), Histoire du sport féminin (165-184). Paris : L'Harmattan.

Robert, P., Aubusson de Cavarlay, B., Potier, M.-L., Tournier, P. (1994). Les comptes du crime: les délinquances en France et leurs mesures. Paris: L'Harmattan.

Roché, S. (1994). Insécurité et libertés. Paris : Seuil.

Roché, S. (1996). La société incivile. Qúest-ce que l'insécurité? Paris: Seuil.

Roché, S. (2001). La délinquance des jeunes, Les 13-19 ans racontent leurs délits. Paris: Seuil.

Roché, S. (2003). En quête de sécurité. Causes de la délinquance et nouvelles réponses. Paris : Armand Colin.

Roumestan, N. (1998). Les supporters de football. Paris: Anthropos.

Roynette, O. (2002). La construction du masculin. Vingtième siècle, 75, 85-96.

Simmel, G. (1908). Secrets et sociétés secrètes. Paris : Circé.

Simourd, L. \& Andrews, D. A. (1999). Délinquance chez les hommes, délinquance chez les femmes - corrélation. Consultée en avril 2005, <http:// www.csc-scc.gc.ca/text/pblct/forum/e06/f061g.shtml>.

Skogan, G. W. (1990). Disorder and decline: Crime and the spiral of decay in American neighborboods. New York: The Free press. 
Tarling, R. (1993). Analysing offending: Data, models and interpretation. London: Her Majesty's Stationery Office.

Testanière, J. (1967). Chahut traditionnel et chahut anomique dans l'enseignement du second degré. Revue française de sociologie, 8, 67-91.

Thébaud, F. (1992). Introduction. In G. Duby \& M. Perrot (ed.), Histoire des femmes, Le XX $X^{e}$ siècle (13-23). Paris: Plon.

Théry, I. (2000, 10 mars). La femme n'est pas homme. Le Monde, 12.

Virgili, F. (2002). L'histoire des femmes et l'histoire des genres aujourd'hui. Vingtième siècle, 75, 5-14.

Wallace, M. (2003). Statistiques de la criminalité au Canada, 2002. Juristat, 23, 1-5.

Wieviorka, M. (1999). Violence en France. Paris: Seuil.

Zimmerman, M. (1987). La violence dans les stades de football: le cas de l'Allemagne fédérale. Revue de droit pénal et de criminologie, 5, 441-463. 